Témoigner Témoigner. Entre histoire et mémoire

Getuigen Revue pluridisciplinaire de la Fondation Auschwitz

$121 \mid 2015$

Violences radicales en scène

\title{
Entretien avec Dorcy Rugamba
}

Interview met Dorcy Rugamba

\section{Christian Biet et Dorcy Rugamba}

\section{(2) OpenEdition}

\section{Journals}

Édition électronique

URL : https://journals.openedition.org/temoigner/3309

DOI : 10.4000/temoigner.3309

ISSN : 2506-6390

Traduction(s) :

Interview met Dorcy Rugamba - URL : https://journals.openedition.org/temoigner/3323 [fr]

Éditeur :

Éditions du Centre d'études et de documentation Mémoire d'Auschwitz, Éditions Kimé

Édition imprimée

Date de publication : 1 octobre 2015

Pagination : 76-83

ISSN : 2031-4183

Référence électronique

Christian Biet et Dorcy Rugamba, « Entretien avec Dorcy Rugamba », Témoigner. Entre histoire et mémoire [En ligne], 121 | 2015, mis en ligne le 01 novembre 2016, consulté le 04 février 2022. URL : http://journals.openedition.org/temoigner/3309; DOI : https://doi.org/10.4000/temoigner.3309 


\section{Dorcy Rugamba}

$\rightarrow$ Entretien mené par Christian Biet le 19 février 2015

(1) Daniel Weyssow, «Rwanda 94. Une tentative de réparation symbolique envers les morts ", Témoigner. Entre histoire et mémoire, $\mathrm{n}^{\circ}$ 117, Paris, Kimé, p. 15.

(2) Saint-Maurice, Da Ti M'beti, 2005
M etteur en scène, auteur et acteur, Dorcy Rugamba étudie au Conservatoire de Liège où il obtient le premier prix d'art dramatique. En 1999, il co-écrit la pièce Rwanda 94, un spectacle de six heures sur le génocide des Tutsi au Rwanda ${ }^{1}$, représenté pour la première fois au festival d'Avignon par la compagnie belge Groupov avec une mise en scène de Jacques Delcuvellerie. La pièce, qui a effectué une tournée mondiale, est couronnée par le prix de la meilleure pièce belge en 2001 et le prix de la Critique en France en 2002. À l'initiative de Dorcy Rugamba, le collectif d'artistes rwandais Urwintore est créé en 2001 dans le but d'impulser une dynamique des arts scéniques au Rwanda et de favoriser les échanges entre les artistes rwandais et étrangers. En 2004, Dorcy Rugamba est engagé par Peter Brook pour jouer le rôle d'Amkoulel dans Tierno Bokar, adaptation du livre d'Amadou Hampâté Ba, Vie et enseignement de Tierno Bokar : Le sage de Bandiagara. En 2005, il publie Marembo ${ }^{2}$ retraçant les derniers jours de sa famille au Rwanda. La même année, il adapte et met en scène L'Instruction de Peter Weiss qu'il représente successivement au Festival Émulation de Liège (Belgique), aux Bouffes du Nord (Paris), au Young Vic Theatre (Londres), au Chicago Shakespeare (Chicago), au Kasser Theater (New Jersey), au Bankart Studio (Yokohama), aux Récréâtrales (Burkina Faso) et au Rwanda, à Butare et Kigali à plusieurs reprises. En 2007, il écrit et joue Bloody Niggers, mis en scène par Jacques Delcuvellerie en coproduction avec le Théâtre National et le festival de Liège.

\section{Pourrait-on dire que, pour vous, « tout commence avec Rwanda 1994 ? "}

Dorcy Rugamba : Non pas du tout. J'ai commencé les arts de la scène tout jeune au Rwanda, sous la direction de mon père, Cyprien Rugamba, avant de fonder ma propre compagnie « Isango » en 1992. Ce qui débute réellement avec Rwanda 1994, c'est le théâtre sur les crimes contre l'humanité. La création de Rwanda 1994 venait au lendemain du génocide, à un moment où quasiment rien n’était dit sur cet événement. Les seuls éléments que pouvait en percevoir un Européen tenaient à une version médiatique, surtout télévisuelle, qui n’offrait qu’un survol du sujet renforçant les clichés auxquels on pouvait facilement le réduire. Créer Rwanda 1994 répond ainsi au constat d'un manque de connaissance de ce qui a eu lieu, y compris pour moi-même qui suis Rwandais. Travailler sur l'œuvre m'a fait prendre conscience que tout un pan de cette histoire récente m'avait échappé, ce qui, plus particulièrement, mène de la première colonisation - l'arrivée des Allemands en 1894, puis la tutelle des Belges et enfin le néocolonialisme français - au génocide, cent ans après. 


\section{VIOLENCES RADICALES EN SCÈNE}

\section{Lorsque La Cantate est jouée indépendamment de}

$R$ wanda 1994, lorsqu'elle en est extraite, sans que l'on ait quatre heures de préparation préalable, l'impact émotionnel est radicalement différent. Cela peut paraître une évidence de le dire ainsi, mais on peut aller plus loin. Notamment, en considérant qu'il a été possible de l'extraire de l'œuvre.

D.R. : On est dans deux temps différents. Par rapport à ce sujet, le public d'aujourd'hui n'est pas le même que celui d'il y a quinze ans. Au moment de la création de Rwanda 1994, on était au lendemain du génocide, non seulement les gens étaient mal informés, mais la réalité même du génocide était contestée, voire niée, aussi bien dans les médias que par la voix officielle, diplomatique. Il fallait à ce moment-là apporter la preuve qu'un génocide s'était vraiment passé, un génocide et non un crime de guerre ou une guerre civile ou une guerre tribale comme certains médias le laissaient entendre. Aujourd'hui, cette reconnaissance a eu lieu, le génocide a été prouvé par différents tribunaux internationaux, il est maintenant reconnu officiellement par les Nations Unies, c'est un fait avéré, même si, effectivement, il subsiste des groupes révisionnistes qui ne changeront

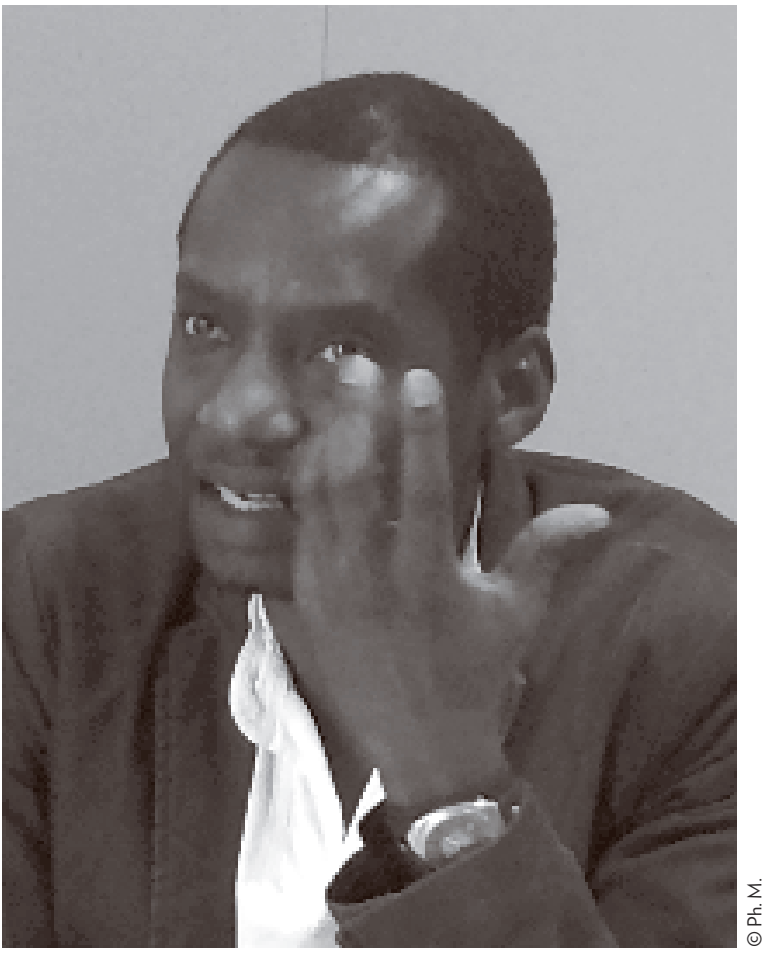
probablement jamais. Malgré ces derniers, la reconnaissance du génocide des Tutsi au Rwanda appartient désormais au domaine public, elle fait partie intégrante de notre savoir commun. C'est pour ça que, en partant de cette connaissance que possède le public aujourd'hui, on peut apprécier la Cantate jouée toute seule sans les autres parties de la pièce qui la précèdent.

\section{Comment définir l'émotion recherchée ?}

D.R. : La Cantate possède une beauté lyrique indéniable. La combinaison du texte très beau et la musique de Garret List portée par les instruments et la voix des cantatrices en fait un morceau d'anthologie. Cependant, ce serait réducteur de n'en faire qu'un chef d'œuvre musical. Ce n'est pas un magnifique drap mortuaire dans lequel on enveloppe cérémonieusement les morts. C'est aussi un poème épique. Qui raconte non seulement l'agonie des victimes des Bisesero, mais aussi la bravoure de ces hommes et ces femmes réduits à rien, n'ayant que leurs mains pour se défendre et qui ont choisi de resserrer les rangs et de se battre. La Cantate est pratiquement la geste guerrière des Bisesero. En cela, elle joue un rôle important dans tout l'édifice de la pièce, car l'un des écueils dans la représentation d'un génocide serait de représenter les victimes comme des agneaux qui vont moutonnement à l'abattoir. Le mot d'ordre de Rwanda 94 est « une réparation symbolique envers les morts, à l'usage des vivants. » Cette dimension symbolique est évidemment importante 
Dorcy Rugamba

(suite)

(3) Rémi Korman, «Rwanda. Les arts et la représentation du génocide des Tutsi », Témoigner. Entre histoire et mémoire, $\mathrm{n}^{\circ} 119$, Paris, Kimé, p. 184-186.

(4) Primo Levi, Si c'est un homme, traduit de l'italien par Martine Schruoffeneger, Paris, Julliard, 1987. pour les rescapés. Car une fois sortis de l'horreur, ils n'ont pas seulement besoin de retrouver la santé et des conditions de vie décentes, mais aussi d'être réhabilités moralement. Symboliquement. La Cantate est un récit digne, comme il y a des images dignes du génocide, qui contrastent avec les campagnes misérabilistes des agences humanitaires. Comme visuels dignes, je pense par exemple à l'œuvre de l'artiste sud-africain Bruce Clarke Les hommes debout ${ }^{3}$.

Il y a une vertu que je trouve dans cette Cantate et qui est liée cette fois-ci à l'interprétation. Le texte est porté par un chœur. La plupart des interprètes ne sont pas Rwandais. La majorité est constituée d'Européens. Ils incarnent pourtant les victimes et s'expriment en leur nom, à la première personne du singulier. Ils disent: « J'étais à tel endroit, à telle date, sur la colline de Muyira. » On n'est pas du tout ici dans une convention de théâtre réaliste. Cela permet d'universaliser le drame. D'en faire littéralement un crime contre l'humanité.

Au moment vous commencez à travailler sur du théâtre documentaire ou sur un autre type de théâtre comme Bloody Niggers, qu'avez-vous appris de l'expérience de Rwanda 1994 ?

D.R.: Justement, la choralité. Le chœur, ça reste une collectivité qui s'exprime. Un génocide atteint la collectivité, c'est un crime où périssent des millions d'individus. Cela dépasse le drame d'un être humain, le chœur alors arrive à ce moment-là, du moins dans la forme d’une représentation théâtrale.

En somme, pour échapper au fait d'être un numéro qui va être abattu, ce que vous proposez, qui n'est pas sans rapport avec les choix de Primo Levi dans l'adaptation théâtrale de Si c'est un homme ${ }^{4}$, ce n'est pas d'être un individu pour résister en tant que numéro, mais d'être à l'intérieur d'un chœur, c'est-à-dire à l'intérieur d'un système qui va résister par le lien qu'il propose dans sa figuration même. Comment avez-vous repris cette forme chorale, comment l'avez-vous rejouée, avec quel texte?

D.R. : J'ai repris la forme chorale avec la mise en scène de l'Instruction de Peter Weiss. Le choix de la pièce de Weiss s'est imposéà moi au début des tribunaux gacaca au Rwanda. Les Gacaca sont des tribunaux populaires mis en place au Rwanda dix ans après le génocide pour désengorger les prisons.

La spécificité du tribunal gacaca est qu'il se tient là où le crime a été commis et que tous les habitants de la localité sont invités à y assister. Les Juges sont des citoyens élus par la collectivité, et les témoins et les accusés s'expriment dans une parole directe sans le filtre d'un juriste. C'est parole contre parole, devant le jury, devant l'assemblée des habitants. En assistant à un procès gacaca, j’ai été frappé tout de suite par un aspect : le fait que ces procès impliquaient tout le monde. Les victimes, les accusés, les témoins, l'ensemble des habitants appelés à s'exprimer sur les faits, à élire des personnes de moralité irréprochable pour rendre justice. Dans ce grand déballage et cette libération de la parole, j’ai vu une forme de théâtre possible. Il se jouait là un drame collectif, englobant victimes, bourreaux, témoins, jurés, la cour, dans un même chœur dont l'expression traduit le drame de la nation rwan- 


\section{VIOLENCES RADICALES EN SCÈNE}

daise. À Butare par exemple, les Gacaca se tenaient les mercredis. Les autres jours de semaine, les gens se comportaient de la même manière, vaquaient aux mêmes activités et adoptaient les mêmes comportements sans qu'on puisse distinguer qui le jour du procès se retrouvera dans le box, qui sera membre de la Cour ou qui se verra accusé par un témoin. Il y avait donc là une collectivité qui a une identité dont l'expression chorale se rompait au moment des procès en plusieurs voix. Et c'est dans cette rupture, que se donnait à entendre toute la dimension du génocide. En termes de théâtralité, rien ne pouvait mieux représenter le drame collectif de la nation rwandaise, que ce chœur qui se brise au moment des Gacaca.

Ce qui m'a amené à L'Instruction de Peter Weiss, qui est une pièce documentaire traitant elle aussi d'un drame national. Du premier procès instruit par un tribunal allemand contre des Allemands pour des crimes commis aussi contre des Allemands : le procès de Francfort en 1964. Il y avait bien évidemment d'autres nationalités parmi les accusés et les victimes, mais cette dimension d'un pays qui se confronte à lui-même est très présente. D’où un parallèle possible avec le Rwanda.

Il y a une autre raison pour laquelle j'ai choisi L'Instruction et surtout de la faire jouer par des acteurs rwandais. Je me suis rappelé qu'avant le génocide au Rwanda, j'avais vu plusieurs productions sur le génocide des Juifs et qu’à aucun moment je ne m'étais senti concerné. Pour schématiser, c'était pour moi une histoire de Blancs, entre Européens. Le genre de chose qui ne pourrait jamais arriver en Afrique. Et cette vision distanciée a continué y compris durant les quatre ans qui ont précédé le génocide, de 1990 à 1994. Années durant lesquelles le discours ouvertement raciste du mouvement Hutu Power ressemblait pourtant de plus en plus à la rhétorique nazie, alors que les pogroms se multipliaient dans le pays. Je me trouvais à cette époque à l'université du Rwanda, à Butare, mais à aucun moment, aucun parallèle ne fut fait entre la situation que nous étions en train de vivre et ce qui s'était passé en Europe dans les années trente et qui avait atteint son paroxysme avec le génocide des Juifs et des Tziganes.

\section{La reconnaissance de l'exceptionnalité du génocide juif vous aurait-elle empêché de voir ce qui vous attendait? \\ D.R. : Ce qui créait plutôt de la distance, c'est que dans les représentations qui} étaient faites, le contexte prenait beaucoup plus de place que le mécanisme même du crime de génocide. C'était du coup plus difficile de comprendre le génocide comme projet politique, comme crime idéologique. Les bourreaux y apparaissaient surtout comme des gens différents, comme si le génocide était un crime typiquement allemand. Ceci m’a amené à penser que représenter un génocide en s'écartant du naturalisme pouvait être intéressant. Et que cela pouvait mettre plus à nu le génocide comme projet politique et comme crime idéologique. C'est la raison pour laquelle dans cette production, tous les acteurs sont Rwandais bien que les personnages soient Européens. L'acteur est ainsi dans une distanciation évidente. C'est le raconteur d'une histoire. Celui qui le regarde ne voit pas un Allemand ni un Africain typique. Le personnage n'est pas emprisonné dans une identité ethnique. 
Dorcy Rugamba (suite)

\section{Dans cette pièce, tous les acteurs jouent des rôles interchangeables, sauf un, n'est-ce pas?}

D.R. : Exactement, sauf un. Il a fallu adapter le texte dans une forme plus proche de la tragédie antique avec un chœur et un coryphée. Pour insister sur la dimension collective du drame, mais aussi pour d'autres raisons. Le texte original de Peter Weiss fait 5 heures à la lecture. C'est une pièce verbatim que Weiss a découpée en onze chants selon une structure qui s'inspire de l'Enfer de Dante. Le texte avance dans l'horreur, et sa progression suit l'évolution des méthodes d'extermination expérimentées par les SS à Auschwitz, chaque seuil fait passer au palier suivant avec des méthodes de plus en plus radicales, comme une descente en enfer jusqu’à la solution finale. Si l'œuvre de Dante est bien connue en Europe, ce n'est pas le cas au Rwanda. Il fallait trouver une autre résonance formelle qui soit proche des références théâtrales là-bas. Pour réduire le texte, un autre critère aussi a guidé l'adaptation. En 1964, lors du procès de Francfort, pour de nombreux témoins, c'était la première fois qu’ils racontaient ce qui leur était arrivé. L’opinion publique ignorait vraiment ce qui s'était réellement passé dans les camps d'extermination. Aussi la pièce de Weiss fourmille-t-elle de détails précis comme dans une véritable instruction judiciaire. De telle sorte que pour le spectateur, la réalité du génocide ne fait aucun doute à la fin de la pièce. Ce long texte était important à ce moment précis de l'histoire, car il apportait la preuve du génocide venant de la parole même des tueurs, de celle des victimes et de témoins impliqués comme les Sonderkommandos. Ce panachage était crucial 20 ans après le génocide. Aujourd'hui, c'est différent. Le public qui vient voir L'Instruction de Weiss au théâtre sait qu'il y a eu un génocide, nul besoin de leur en fournir la preuve. Par contre et c'est là que l'adaptation s'avérait nécessaire, plus l'information factuelle du génocide des Juifs est partagée par un plus grand nombre, moins il y a de gens qui en ont une connaissance empirique. Qui peuvent réaliser réellement ce qu'est un génocide. Quel type de crime est un génocide et qu'est-ce qui le distingue d'autres tueries. L'adaptation et la mise en scène ont donc pour but d'éclairer le spectateur sur la nature du crime. Donner à entendre, à travers les paroles des rescapés des camps, ce qu'est l'acte d'anéantissement. Éclairer le public à travers les paroles des accusés dans le prétoire, sur quoi porte un crime idéologique. Comment le tueur justifie-t-il le geste qu'il a commis ? Comment argumente-t-il ? Alors, le crime émerge. Les acteurs au cœur du processus évitent de réduire le criminel à une brute épaisse, de le caricaturer. C'est peut-être même un bon père de famille, qui sait? Ce qui nous intéresse c'est ce qui se passe dans sa tête, ce qui guide sa main et ce qui peut se transmettre, qu'il peut enseigner, propager...

Dans cette pièce, votre façon de faire se déplacer la question entre ce qui se passe dans la tête des bourreaux et dans celle des victimes est impressionnante. Comment cela prend-il forme au niveau de l'écriture et de la mise en scène ?

D.R. : La forme elle-même d'une instruction judiciaire permet d'échapper à une écriture et une mise en scène qui ressembleraientà une messe solennelle de condamnation morale du crime. L'investigation permet de donner la parole à toutes les parties. J'essaie toujours de me mettre à la place du spectateur, de me dire : mais si 


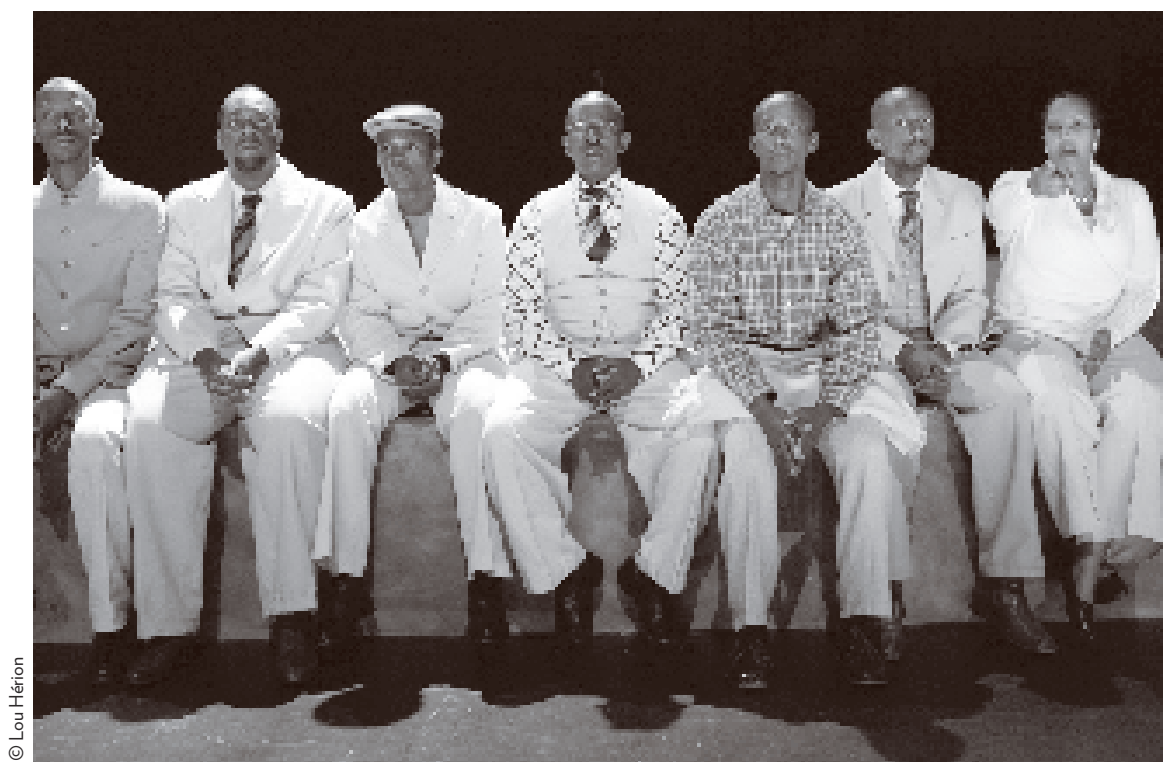

quelqu’un arrive pour me faire la leçon, est-ce que je la reçois ou non? Il est important que l'expérience que l'on propose au spectateur engage ses capacités de jugement. Par exemple, parmi les accusés que Weiss a retenus, il y a un jeune prévenu qui s'appelle Starck, qui était très jeune à l'époque des faits. Un jeune SS qui sortait des jeunesses hitlériennes. Dans le camp, Starck s'occupait entre autres de sélectionner les détenus à la rampe d'arrivée des trains, et de les classer en trois catégories. Les prisonniers politiques, les droits communs et les prisonniers raciaux. À la question du Juge : « Est-ce que vous trouviez normal d'envoyer des femmes et des enfants dans les chambres à gaz? », Starck répond « Oui. À l'époque, il y avait un principe de responsabilité raciale collective. » Pour le spectateur, cette parole du tueur permet de mieux prendre conscience de l'abomination du crime que la simple formulation du «plus jamais ça », car elle donne à voir non seulement le crime et l'exécutant, mais aussi l'idéologie dont il se sert.

Dans ma pièce Bloody Niggers, c'est également à cela que j'ai voulu m’attaquer. Je voulais partir de 1994 au Rwanda et faire une généalogie du génocide. Par quel chemin parcouru par l'humanité en sommes-nous arrivés là? Pour cela,j’ai exhumé plusieurs crimes contre l'humanité pour les porter à la connaissance du public, mais les exposer en soi ne suffit pas. C'est leur justification par les auteurs qui permet de les éclairer d'un jour nouveau. On peut, par exemple, regarder des documentaires sur la colonisation, prendre connaissance de l'extermination, parfois totale, de peuples - comme ce fut le cas pour les Tasmaniens au XIX siècle - en être ému aux larmes, mais on n'en saisit réellement l'horreur qu'en découvrant l'ampleur du discours de justification de ces crimes, porté non par des idéologues racistes confirmés à la 
Dorcy Rugamba

(suite)

(5) Performance montée par le metteur en scène sud-africain blanc Brett Bailey. Ce spectacle déambulatoire propose au spectateur de sarrêter devant 12 tableaux "vivants », où des Noir[e]s sont mis en scène dans des décors similaires à ceux des zoos humains du début du $X X^{\mathrm{e}}$ siècle. L'idée est d'établir un parallèle avec la situation actuelle des migrants d'Afrique subsaharienne qui constituent l'autre partie des tableaux « vivants ».
Gobineau, mais par des philosophes humanistes, des hommes d'Église, des juristes et des scientifiques tout à fait reconnus et respectables. C'est là, dans les mots, dans le projet, que la nature totalitaire de l'impérialisme colonial apparaît dans sa démesure.

Effectivement, vous passez de la question du génocide rwandais et du génocide des Juifs d'Europe à celle du colonialisme et récemment vous avez pu vous exprimer à propos de la traite dans Exhibit $B^{5}$. Peut-on, pour vous, parler de génocide à propos de la traite? Et peut-on en faire du théâtre?

D.R. : Dans Bloody Niggers j'ai consacré tout un chapitre à la traite négrière et au commerce triangulaire que je considère de même nature que les crimes de génocide. Car la traite transatlantique diffère de tous les autres types d'esclavage ou de servitude qui ont existé ou existent encore dans le monde. Encore une fois, la distinction est dans l'idéologie. Ailleurs, en d'autres temps ou sous d'autres cieux, l'esclave est un captif, un homme privé de liberté, mais un être humain. Dans les colonies d'Amérique, la qualité d'être humain lui est contestée juridiquement dans les textes de loi, par les scientifiques qui tentent d'en fournir la preuve, par les théologiens qui statuent qu'il n'a pas une âme, etc. Concrètement dans les faits comme sur le plan des principes, il est traité comme du bétail. «L'esclave est un bien meuble », stipule l'article 44 du Code Noir. C'est un acte d'anéantissement dont la portée idéologique s'appliquait au-delà des plantations et disqualifiait l'ensemble des hommes noirs.

C'est évidemment une matière très sensible à traiter au théâtre, car comme tous les crimes contre l'humanité, la traite a un aspect spectaculaire qui peut sidérer. Le risque devient alors trop grand quand on le met en scène d'en faire une attraction et de transformer le spectateur en voyeur malgré lui. C'est le travers, à mon humble avis, dans lequel est tombé Exhibit B.

Précisément, quel rapport entretenez-vous avec les formes conventionnelles dramatiques du théâtre, ce dont justement un certain nombre de pièces du théâtre français ne se dégagent pas?

D.R. : Je n'ai rien contre les conventions dramatiques, même si je pense qu'il faut réinventer des formes et toujours les questionner. Je pense vraiment au public, que peut-on lui proposer pour appréhender la violence? Ne lui proposer que des coups et des litres d'hémoglobine, c'est considérer qu'il ne peut approcher la violence que par les moyens les plus élémentaires, alors que cette violence est beaucoup plus complexe.

Ne peut-on pas saisir violemment par l'image, non pour sidérer, mais pour donner lieu à la pensée ? Notamment avec l'image sanglante de la figuration du bourreau en action. Autrement dit, en position de voyeur, culpabilisant, voire éprouvant un certain plaisir, est-on malgré cela dans l'incapacité de penser?

D.R. : Il est difficile de dire qu'il faut le faire, ou le contraire. L'art de la représentation est un processus complexe. Ma question n'est pas qu'est-ce que j'ai le droit de montrer ou pas, mais comment puis-je le faire, dans quelle forme, avec quels 
moyens. Si je choisis par exemple de mettre en scène un acte de viol comme il y en a souvent dans les crimes de masse. Il n'y a rien de mal a priori à faire cela. Par contre, si par des moyens de mise en scène, je choisis d'érotiser à l'extrême cette scène de viol, j’amène le spectateur à porter sur la victime le même regard que le violeur. Ça veut dire que je suis dans la complaisance avec le crime. Je me délecte alors d'un acte que je prétends condamner. Les victimes pourraient légitimement dans ce cas se sentir avilies et dénoncer un spectacle indigne. C'est ça au fond la question. Dans les moyens que je m'autorise en montrant des images violentes, dois-je solliciter ce genre de stimuli? C'est une question d'ordre esthétique, or l'esthétique... on ne peut pas en parler en termes absolus.

Une dernière question : le théâtre consiste-t-il à mettre à nu les mécanismes des événements, de l'histoire, de ce qui a eu lieu en y ajoutant de l'émotion ?

D.R. : Oui, bien sûr, il faut qu'il y ait de l'émotion. En tout cas, il faut du sensoriel, il ne peut pas y avoir que des stimuli intellectuels. Donc, il faut pouvoir attraper le spectateur par les différentes portes de la perception, le théâtre, ce n'est pas une conférence, on ne peut pas seulement y délivrer un discours. Le théâtre, c'est une expérience. C'est vraiment le fond de l'affaire pour moi. Le théâtre doit mettre à nu les mécanismes qui sont en jeu. Qu'est-ce qui se cache derrière le masque? Qu'estce qui travaille effectivement les forces en place qui vont décider de l'assentiment d'une population? 